\title{
WHICH HORSES ARE MOST SUSCEPTIBLE TO THE INITIAL NATURAL TRAINING?*
}

\author{
Iwona Janczarek, Anna Stachurska`, Izabela Wilk \\ Department of Horse Breeding and Use, \\ University of Life Sciences in Lublin, Akademicka 13, 20-950 Lublin, Poland \\ •Corresponding author: anna.stachurska@up.lublin.pl
}

\begin{abstract}
The objective of the study was to estimate the horses' susceptibility to the initial natural training by one mark regarding both the time of training and the heart rate, as well as to verify whether the time of internalizing a task and the heart rate are strictly correlated. The material included 69 Thoroughbred, Purebred Arabian and Angloarabian horses. Three-day training consisted of consecutive stages-tasks: the concentration on the trainer, desensitizing, preparation for saddling, and saddling. The individual training times and heart rates were classified into three kinds of sections: low, intermediate, and high. The breeds were scored on a three-point scale according to the number of representatives in a section. Pearson's correlations for particular tasks were found between the data in the training time sections and the heart rate. The estimate resulting from the study demonstrates that Thoroughbreds are the most susceptible to the natural training. Purebred Arabians rank second and Angloarabians rank lowest. The Angloarabians need more time to internalize the training tasks. The short time of internalizing a task by the horse is negatively correlated with the heart rate. However, in horses which need a longer time for the training, the HR is often heightened as well. That suggests the training time should be adjusted to the level of emotional arousal in a horse.
\end{abstract}

Key words: horse, training time, heart rate, natural training method

Already Xenophon ca. 400 BC suggested that horses should be willing to cooperate with the rider. Nowadays, the desired horse most often used in many parts of the world in leisure, tourism, equestrian sports or racing, has been defined as a happy companion. To comply with this criterion, the training of the horse cannot disturb its emotionality and proper interaction with the human. The horse's susceptibility, i.e. the responsiveness to given tasks in terms of facility of learning and stress reaction, is crucial for the effectiveness of the training. The natural horsemanship becomes

\footnotetext{
*This study was supported by the Ministry of Science and Higher Education, grant No. N N311 502039.
} 
more and more popular, since assumptions of the school focus on training the horse in agreement with its nature. In consequence, the human wins confidence of the horse. More profitable effect of the natural horsemanship compared to conventional training methods was proved (Visser et al., 2009; Kędzierski et al., 2012). The trainers apply body language and negative reinforcement when schooling the horse to avoid pressure. In turn, the approach and retreat techniques are to be understood by the horse as an invitation or chasing out of the herd led by the trainer (Waran et al., 2007). All those methods contribute to shortening the time of the training and lowering the horse emotional arousal (Kędzierski et al., 2012). The horse reactivity affects the process of learning (Valenchon et al., 2013). According to Calabrese (2008), the low emotional arousal during schooling is crucial to achieve favourable effects. The role of the heart work in the regulation of cognitive processes in humans has been known for a long time (Thayer and Lane, 2009).

The horse's susceptibility to schooling also depends on its individual characteristics associated with the breed and the sex (Lloyd et al., 2008; Duberstein et al., 2010; Górecka-Bruzda and Jezierski, 2010; Janczarek et al., 2013 b). As is commonly known, the horse breeds largely differ with regard to both physical and psychical characteristics. Hausberger et al. $(2002,2011)$ found that Angloarab horses showed friendly attitude towards human and passed the bridge test more rarely than French Saddlebreds. Hausberger et al. (2004) revealed that of 16 horse breeds, Purebred Arabians and Thoroughbreds showed the highest level of reactivity during the bridge test. In the bridge test, a horse is led using a halter to cross a bridge, i.e. an unknown obstacle. Lloyd et al. (2012) also demonstrated that Purebred Arabians and Thoroughbreds were more excitable but simultaneously more sociable and inquisitive. In our earlier studies on the horse's response to natural training methods performed on another group of horses, we demonstrated that compared with Thoroughbreds and Purebred Arabians, halfbred Angloarabs needed more time to complete training stages and their heart rate was heightened (Janczarek et al., 2013 b). However, from less pronounced differences between Thoroughbreds and Purebred Arabians, we could not infer which of the two breeds better reacted to the natural training.

The evaluation of the susceptibility to the training in different horse breeds is important in the context of the breed purpose, methods of training and using. To date studies show that the complex assessment of the horse's susceptibility to the training is difficult. The judgment usually consists of measuring the time of internalizing particular tasks by the horse, the level of the emotional arousal as well as scoring the horse's behaviour during the training. The results of such approaches are presented in different measures and concerning the natural training, correlations between them are not known. As mentioned, in our previous investigation we measured the response to the initial natural training with the length of the time necessary to complete a stage of the training and with the horse's heart rate during the stages (Janczarek et al., 2013 b). The analysis of variance gave plenty of results sometimes difficult to interpret. This time we aimed at estimating the horses' susceptibility to the initial natural training by one mark regarding both the time of training (TT) and the heart rate (HR) simultaneously. Such a total mark would show which horse group more quickly internalizes the tasks of the training and simultaneously is less emotionally 
aroused. The analysis was exemplified in three breeds. We also aimed at verifying whether the time of internalizing a task and the heart rate in the initial natural training of horses are strictly correlated. Such a correlation would make it possible to apply only one parameter in training practice.

\section{Material and methods}

The material included 23 Thoroughbred (TB), 23 Purebred Arabian (PA) and 23 halfbred Angloarabian (AA) clinically sound, naïve horses. In each breed group there were 11 to 12 fillies and 11 to 12 colts. The PA and AA horses were examined in Janów Podlaski Stud and the TB horses in two private studs. The TBs were approx. 18 months old, whereas PAs and AAs were approx. 30 months old. that is at the age of the beginning of the training before first races. The horses were not trained before the experiment and they did not know the round-pen. The contact with humans consisted of everyday management, periodic prophylactic treatment, as well as hoof trimming. The experiment took place in autumn. After the experiment, the horses were taken to the racing track in order to begin the specialist training. The initial training in a round-pen was conducted by a licensed trainer of the natural horsemanship. The trainer brought the horse into the round-pen and taught to avoid pressure, to move in a long line, as well as familiarized the horse with novel objects. According to individual horse training performance, different cues were used in different order and repetition during each of the training sessions. The training sessions were performed on three successive days during 30-60 min each day. The training consisted of consecutive stages-tasks: horse concentration on the trainer, desensitizing, preparation for saddling, saddling, preparation for mounting, and mounting. In the study, the following tasks in which all the horses succeeded on a particular day were considered: the concentration and desensitizing on the first day, additionally the preparation for saddling on the second day and additionally the saddling on the third day.

We assumed that the horses were susceptible to the training when the high effectiveness of the training expressed with the short TT was associated with slight emotional reaction expressed with the low HR. The individual TT needed for the horses to accomplish the tasks was measured with a hand stopwatch to an accuracy of one second. The stopwatch was synchronized with the telemetric $810 \mathrm{~S}$ transmitter put around the horse chest to register the HR in five-second intervals (bpm - beats per minute). On each day, the TT and HR were measured since entering the horse into the round-pen to the end of the training. Sequences of mean TTs and HRs in consecutive tasks on successive training days registered in the experiment were ranked and divided according to the mean and standard deviation into three kinds of sections:

- desired low sections $->($ mean $-\mathrm{SD})$;

- intermediate sections $-\geq($ mean $-\mathrm{SD})<($ mean $+\mathrm{SD})$;

- undesired high sections $-\geq($ mean $+\mathrm{SD})$. 
The sections grouped horses most susceptible to training, medium and least susceptible. Firstly, such a division enabled studying the correlations in detail (Janczarek et al., 2013 c). Sometimes in a low section a correlation may be positive and in a high section the same correlation can be negative. Thus, the traits considered in total may seem not to be correlated. Secondly, the division into the sections made it possible to combine the TT and HR parameters into one parameter: the susceptibility. The breeds were ranked with regard to the number of representatives in a section: a breed of a highest number of representatives scored three points, a breed at an intermediate position scored two points and at a third position scored one point. In the case of equal numbers of horses of two or three breeds in a section, the breeds were awarded the same joint scores, e.g. 1.5 instead of twice 2. The total score for the TTs, for HRs as well as for the combined susceptibility parameter in the sections, was the final evaluation of the horse breed.

The TT and HR means were compared by multifactorial analysis of variance (ANOVA GLM) using SAS software (2003). The following factors were considered: the breed (TB, PA, AA), sex (colts, fillies), and training day $(1,2,3)$. In the further analysis we focused on the breed and training day factors. The significance of differences in means was estimated with Tukey's test and accepted at the level of $\mathrm{P}<0.05$. Pearson's correlations for particular tasks were found between the data in the TT sections and the HR. The TT sections were chosen since the time parameter and the TT sections would be easier to apply in practice than the HR sections. The distributions of the scores in the sections for particular breeds were compared with $\chi^{2}$ test.

\section{Results}

As shown in Figure 1, the TT necessary to concentrate on the trainer and desensitizing was lowering on the successive days of the training. The preparation for saddling took similar time on the second and third days. The HR during the concentration and desensitizing was significantly higher on the first day than on the third day, while during the preparation for saddling it was alike on the second and third days. It can be noted that the TT in the tasks began for the first time was the longest for desensitizing, shorter for the concentration, then for the preparation for saddling and the shortest for the saddling. As to the HR, the order was the following: the concentration, desensitizing, saddling, and preparation for the saddling.

Significant high correlations between the TT sections and HR occurred rarely (Table 1). Significant negative correlations were found on the second and third training days during the concentration in the low sections. Significant correlations also concerned the saddling on the second training day, although in the low sections the correlation was negative, whereas in the intermediate and high sections the correlations were positive. Insignificant correlations were also usually negative in the low sections and positive in the high sections. 

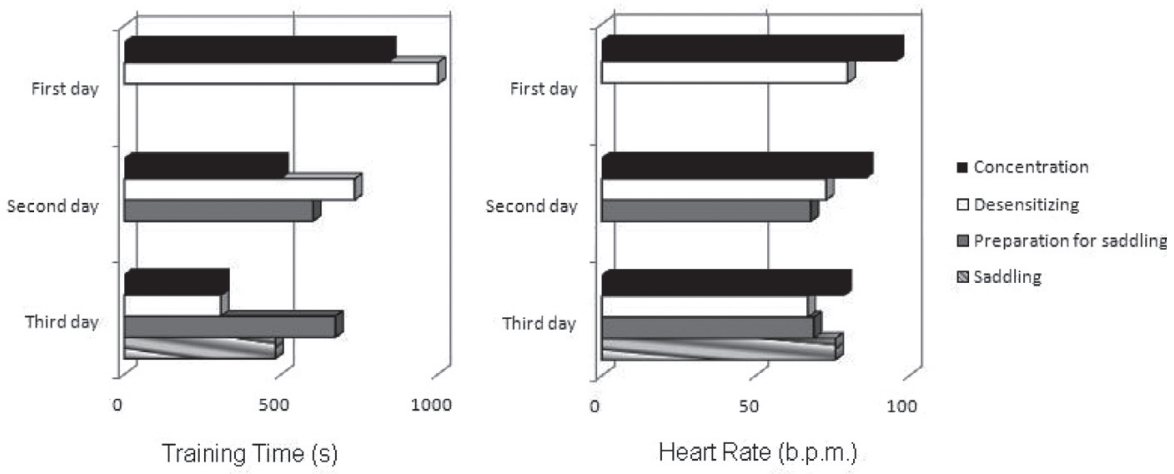

Figure 1. Training Time (s) and Heart Rate (bpm) in particular tasks on successive training days. The following means differ minimum at $\mathrm{P} \leq 0.05$ : Training Time in concentration (between all days), Training Time in desensitizing (between all days), Heart Rate in concentration (between first and third days), Heart Rate in desensitizing (between first and third days)

Table 1. Correlations between Training Time (in sections) and Heart Rate in particular tasks on successive training days

\begin{tabular}{|c|c|c|c|c|}
\hline $\begin{array}{l}\text { Tasks } \\
\text { Training } \\
\text { Time sections }\end{array}$ & Concentration & Desensitizing & $\begin{array}{l}\text { Preparation } \\
\text { for saddling }\end{array}$ & Saddling \\
\hline \multicolumn{5}{|c|}{ First training day } \\
\hline Low & -0.247 & 0.407 & - & - \\
\hline Intermediate & 0.144 & 0.115 & - & - \\
\hline High & -0.018 & 0.429 & - & - \\
\hline \multicolumn{5}{|c|}{ Second training day } \\
\hline Low & $-0.847^{*}$ & -0.107 & 0.208 & $-0.562 *$ \\
\hline Intermediate & 0.242 & -0.024 & -0.026 & $0.433 *$ \\
\hline High & 0.177 & 0.488 & 0.439 & $0.596^{*}$ \\
\hline \multicolumn{5}{|c|}{ Third training day } \\
\hline Low & $-0.567^{*}$ & -0.462 & 0.420 & -0.239 \\
\hline Intermediate & 0.115 & 0.239 & 0.191 & 0.231 \\
\hline High & 0.015 & 0.540 & 0.044 & 0.076 \\
\hline
\end{tabular}

*Correlations significant at $\mathrm{P} \leq 0.05$.

Table 2 presents the ranges established for the sections for the TT and HR. Breeds classified within those ranges according to the frequency of representatives in particular tasks on successive days are shown in Figures 2-4. The prevalence of the TBs is visible in the desired low sections in the successive tasks, particularly in the concentration. The PAs were more numerous in the TT of concentration on the first training day, desensitizing on the first and third days as well as preparation for saddling on the third day. The PAs' HR was the most frequently the lowest only during the saddling. In the intermediate sections, the PAs dominated on the first two days. whereas on the third day the AAs were the most frequent. The AA's prevalence was distinct in the high, undesired sections on all the three days. The TB and PA horses 
scored highest only in $5.5 \%$ and $16.6 \%$ of high sections, respectively. No tendency in the breed scores corresponding with the kind of the task was observed.

Table 2. Training Time (s) and Heart Rate (bpm) sections

\begin{tabular}{|c|c|c|c|c|}
\hline Sections & Concentration & Desensitizing & $\begin{array}{l}\text { Preparation } \\
\text { for saddling }\end{array}$ & Saddling \\
\hline \multicolumn{5}{|c|}{ Training Time } \\
\hline \multicolumn{5}{|c|}{ First training day } \\
\hline Low - below: & 295 & 397 & - & - \\
\hline Intermediate - within: & 295-1393 & $397-1619$ & - & - \\
\hline High - above: & 1393 & 1619 & - & - \\
\hline \multicolumn{5}{|c|}{ Second training day } \\
\hline Low - below: & 172 & 136 & 218 & - \\
\hline Intermediate - within: & $172-830$ & $136-1332$ & $218-986$ & - \\
\hline High - above: & 830 & 1332 & 986 & - \\
\hline \multicolumn{5}{|c|}{ Third training day } \\
\hline Low - below: & 132 & 74 & 140 & 263 \\
\hline Intermediate - within: & $132-494$ & $74-543$ & $140-1206$ & $263-700$ \\
\hline High - above: & 494 & 543 & 1206 & 700 \\
\hline \multicolumn{5}{|c|}{ Heart Rate } \\
\hline \multicolumn{5}{|c|}{ First training day } \\
\hline Low - below: & 71 & 59 & - & - \\
\hline Intermediate - within: & $71-122$ & 59-101 & - & - \\
\hline High - above: & 122 & 101 & - & - \\
\hline \multicolumn{5}{|c|}{ Second training day } \\
\hline Low - below: & 63 & 54 & 52 & - \\
\hline Intermediate - within: & $63-110$ & $54-93$ & $52-84$ & - \\
\hline High - above: & 110 & 93 & 84 & - \\
\hline \multicolumn{5}{|c|}{ Third training day } \\
\hline Low - below: & 57 & 51 & 53 & 62 \\
\hline Intermediate - within: & $57-101$ & $51-83$ & $53-85$ & $62-91$ \\
\hline High - above: & 101 & 83 & 85 & 91 \\
\hline
\end{tabular}

Regardless of the training day and the task, the distribution of the scores for the TT was similar in the breeds ( $\mathrm{P}>0.05$; Table 3 ). However, the TB horses' scores in the low sections were 1.5 times as high as in the high sections, while the AAs scores in the high sections were 2.3 times as high as in the low sections.

The differences in the distribution of the scores for the HR and for the total scores were significant $(\mathrm{P}<0.01)$. The TB horses of a low HR were the most frequent, the PAs were ranked second, whereas the AAs scored the least: 2.7 times as low as the TBs and 1.9 times as low as the PAs. In the high sections, the frequency of AAs of a high HR were 1.5 times as high as in the other breeds. Considering the total scores of the TT and HR in the low sections, the order of the breed scores was the following: the TB, PA and AA. In the high sections the order was opposite, although the TB and PA scores did not considerably differ among each other, simultaneously being 1.6 times as high as the AA scores. 


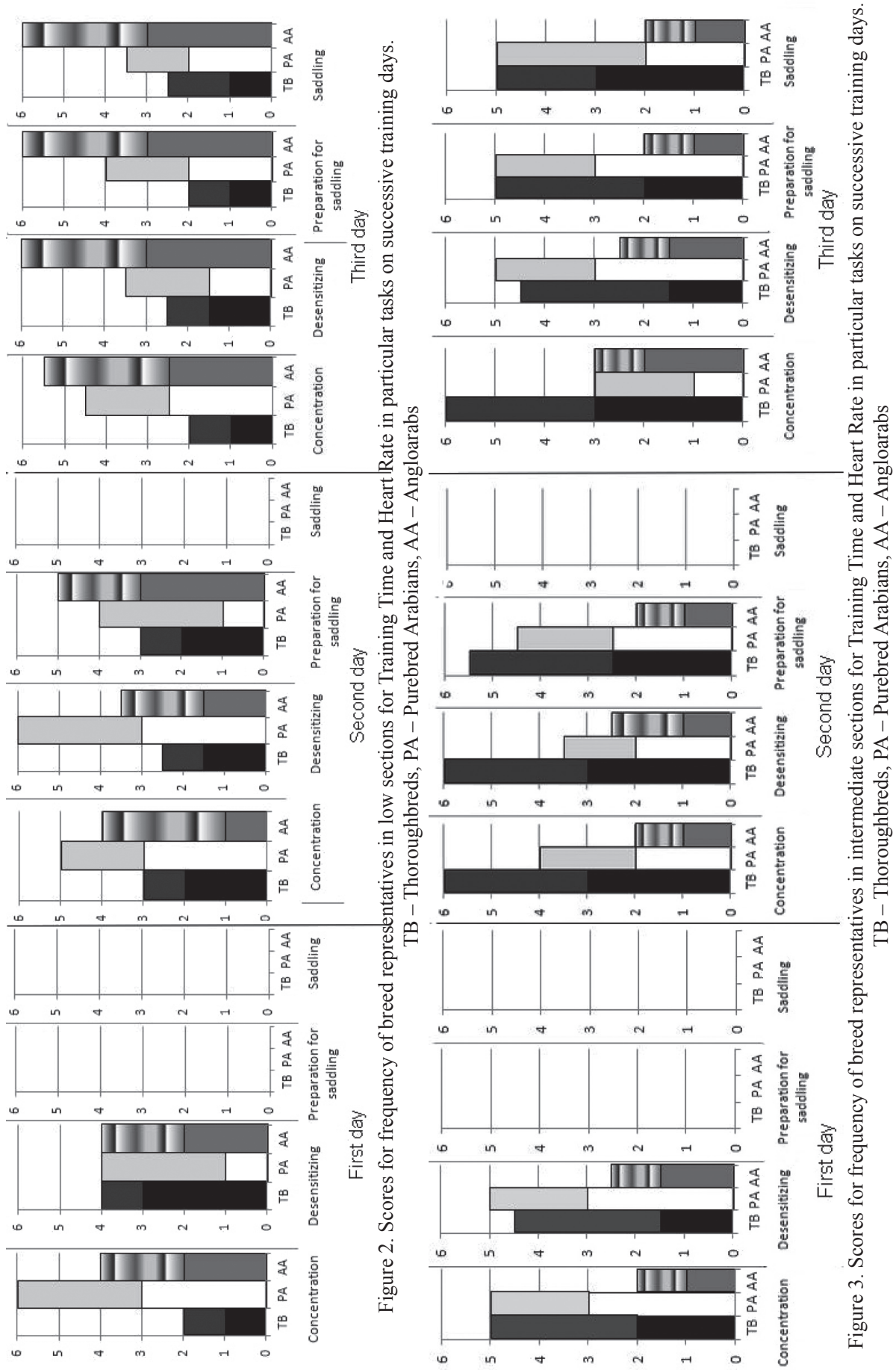




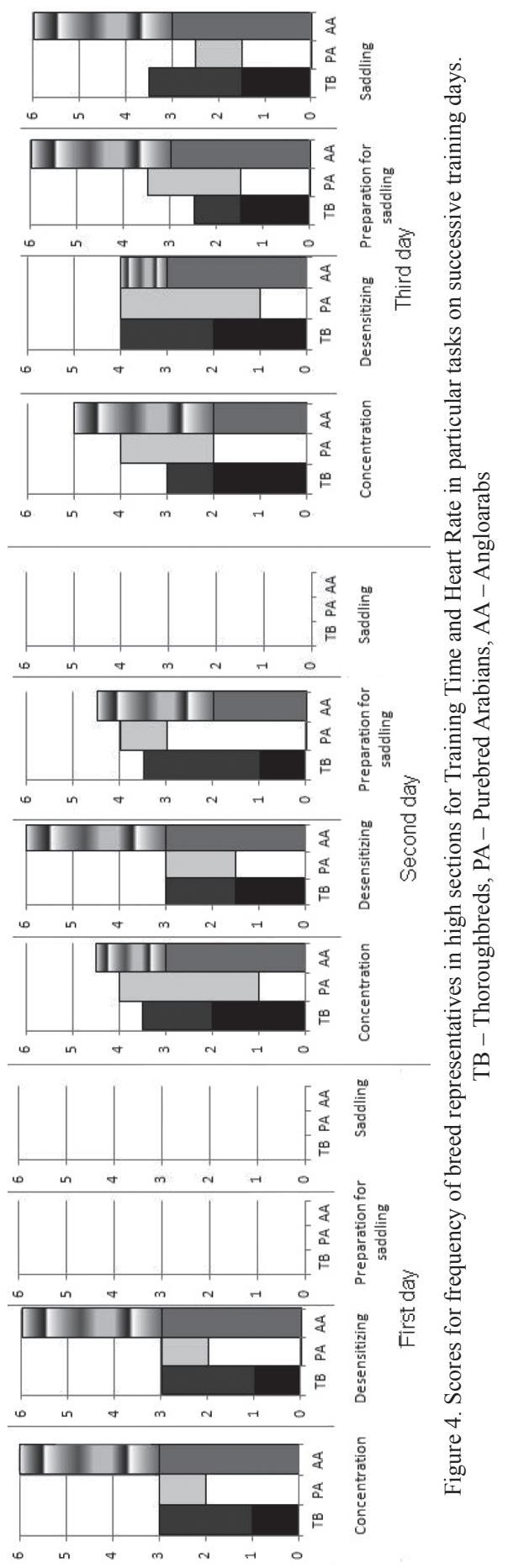


Table 3. Total score for frequency of breed representatives in sections for particular breeds for Training Time, Heart Rate and Susceptibility in all of the tasks and in all of the training days

\begin{tabular}{l|ccc|c|c|c|c|c|c}
\hline \multirow{2}{*}{ Breeds } & \multicolumn{3}{|c|}{ Training Time } & \multicolumn{3}{c|}{ Heart Rate } & \multicolumn{3}{c}{ Susceptibility } \\
\cline { 2 - 10 } & low & $\begin{array}{c}\text { inter- } \\
\text { mediate }\end{array}$ & high & low & $\begin{array}{c}\text { inter- } \\
\text { mediate }\end{array}$ & high & low & $\begin{array}{c}\text { inter- } \\
\text { mediate }\end{array}$ & high \\
\hline TB & 21.5 & 14.0 & 13.5 & 26.0 & 9.5 & 15.5 & 47.5 & 23.5 & 29.0 \\
PA & 21.5 & 19.0 & 15.5 & 18.5 & 21.5 & 15.5 & 40.0 & 40.5 & 31.0 \\
AA & 11.0 & 21.0 & 25.0 & 9.5 & 23.0 & 23.0 & 20.5 & 44.0 & 48.0 \\
\hline
\end{tabular}

Significant differences within distributions for Heart Rate scores and for Susceptibility scores $(\mathrm{P} \leq 0.01)$.

\section{Discussion}

The ground for the proper horse training is the individual approach to each animal and the destination of appropriate time for the most difficult tasks (Waran et al., 2007). At the start of the training, it is also important to begin each training session with a repetition of the tasks which were accomplished on the previous day (Murphy and Arkins, 2007). Thus, the horse internalizes the tasks and the trainer can exclude a coincidence in the horse's reaction (Thomas, 1986). According to Schmidt et al. (2010), when the horse is properly prepared, the critical tasks, i.e. the saddling and mounting by the rider are more easily accepted. Christensen (2013) found that excessive shortening of the habituation might cause a strong stress reaction. The results of the present study show that the TT needed for the concentration and desensitizing distinctly shortens on consecutive training days. The changes of the HR are similar to the TT, though less distinct. As expected, the horse's reaction to the same task was weaker on the successive training days. The lowering HR and TT indicate that horses successively internalized the concentration and desensitizing. According to Janczarek et al. (2013 a), first minutes of the training when the trainer focuses the horse's attention on himself, are particularly exhilarating for both the horse and the trainer. In the present study, it turned out that the TT and HR in the preparation for saddling did not change on the third day compared to the second day. That suggests the task is more difficult to be learned than concentration and desensitizing. The mean HR on the third day shows that the successive tasks did not cause an elevated response until the saddling which increased the parameter. The latter indicates that the saddling is particularly exhilarating, which agrees with earlier findings by Fowler et al. (2012).

The correlations between the TT sections and HR show that the estimation of the horse's susceptibility to the natural training exclusively on the basis of one of the parameters is not precise. Significant correlations between the parameters studied are rare and occur solely on some days of the training, in some sections of the parameters and during some training stages. Hence, analysing the data without the division into sections would not be justified and presumably would not show any significant correlations.

On the second and third days. the correlations were usually negative in the low TT sections and positive in the high TT sections. Such results indicate that the short 
TT tends to be correlated with the elevated HR response, i.e. in horses which internalize the tasks more quickly, the HR is heightened. On the other hand, when the TT is high, the HR can also be heightened, i.e. in horses which need more time for the training, the HR is often high as well. That proves the rules applied in the natural horsemanship to condition the time of the training on the intensity of the horse's emotional reaction. It seems particularly important to properly adapt the time of the saddling to the horse excitability. A too long time of the saddling may negatively influence the emotional arousal. The results document that it is not possible to apply only one parameter when estimating the intensity of the horse's response to the natural training. The trainer should know whether the horse trained belongs to the group of best, intermediate or worst performers in terms of the facility of learning. and within the section should look for the balance between the TT and the HR.

The division of the data into the desired, intermediate and undesired sections of the TT and HR enabled a detailed analysis of the breed influence on the horse susceptibility to the natural training. The results indicate that the breed differences in the training effectiveness measured by the TT and the emotional reaction expressed by the HR, are not strictly associated with the kind of the task, i.e. the stage of the training. The rankings of the breed scores on particular days in different tasks did not show a distinct tendency. That means the total results are informative being based on the consistent material.

The TB horses were the most frequent in the desired HR sections which indicates their reaction to the training is calm. Regarding the TT, they were like the PAs. The results to some extent agree with our earlier findings (Janczarek et al., $2013 \mathrm{~b}$ ). In the cited study. Thoroughbreds and Purebred Arabians showed positive similar response to the initial natural training, whereas Angloarabian horses reacted less favourably. The response was measured among others by the percentage of horses which were successfully saddled and mounted during three days of the training. The results of the present study demonstrate the distinct dominance of the TBs estimated by the total susceptibility to the training, in spite of the fact that when considering solely the TT, the TBs and PAs were similar. Hayes (1998) classified the Thoroughbreds as quite quickly learning but simultaneously difficult to concentrate. Our results do not agree with that finding, particularly regarding the HR. As is known, the HR influences the concentration (Thayer and Lane, 2009; Janczarek et al., 2013 b). Presumably, various training methods differently affect the HR in particular breeds. The natural training technique seems to be an appropriate tool which soothes the TB horse excitability. The low TB reactivity in the initial training noted in the study was opposite to common opinions on the high excitability of that breed (Loyd et al., 2008; Mc Greevy and Thomson, 2006).

The AAs slightly surpassed in numbers the PAs in the intermediate sections, whereas distinctly dominated in the high sections. In the intermediate sections the AAs' frequency became higher than in other breeds on the third training day which shows the AAs need a longer time for schooling and their reactivity is longer heightened. Such results provide evidence for the worse AA properties which were earlier found by Hausberger et al. $(2002,2011)$ in the bridge test and Janczarek et al. (2013 b) in the natural training. Summing up, desired effects of natural training tech- 
nique, measured by the TT, HR and the total susceptibility estimate may be expected in Thoroughbreds. Medium effects should be visible in Purebred Arabians, whereas worse results can be predicted for Angloarabs.

In this study we analysed the susceptibility to the training of horses of different breeds, although a similar analysis comparing horses of various sexes or ages would be equally justified. The comprehension of the responsiveness of different groups of horses to the training methods is of prime interest in practice. Fast learners of moderate HR reaction are particularly desired. In conclusion, the study shows that the quantitative estimation of the horse breed susceptibility to the natural training should be detailed in order to reveal which training conditions for which horses are particularly convenient. The combined evaluation of the TT and HR may lead to other conclusions than according to either parameter analysed alone. The estimate resulting from the study demonstrates that Thoroughbreds are the most susceptible to the natural training. Purebred Arabians rank second and Angloarabians rank lowest. The Angloarabians need more time to internalize the training tasks. The short time of internalizing a task by the horse is negatively correlated with the heart rate. However, in horses which need a longer time for the training, the HR is often heightened as well. That suggests the training time should be adjusted to the level of the horse emotional arousal.

Animal care and experimental procedures were in accordance with the European Commission regulations for the protection of experimental animals and approved by the second Local Ethics Review Committee for Animal Experiments at the University of Life Sciences in Lublin, Poland (Act 2/2010, 19.01.2010).

\section{References}

C a la bre s e E.J. (2008). Stress biology and hormesis: the Yerkes-Dodson law in psychology - a special case of the hormesis dose response. Crit. Rev. Toxicol., 38: 453-462.

$\mathrm{Christensen} \mathrm{J.W.} \mathrm{(2013).} \mathrm{Object} \mathrm{habituation} \mathrm{in} \mathrm{horses:} \mathrm{The} \mathrm{effect} \mathrm{of} \mathrm{voluntary} \mathrm{vs.} \mathrm{negatively} \mathrm{rein-}$ forced approach to frightening stimuli. Equine Vet. J., 45: 298-301.

Duberstein K.J., Gilkes on J.A. (2010). Determination of sex differences in personality and trainability of yearling horses utilizing a handler questionnaire. Appl. Anim. Behav. Sci., 128: $57-63$.

F ow 1 e r V.,. K e n n e d y M., M a r 1 in D. (2012). A comparison of the Monty Roberts technique with a conventional UK technique for initial training of riding horses. Anthrozoos, 25: 301-332.

Góre cka-Bruzda A., Jezierski T. (2010). Breed differences in behaviour related characteristics of stallions evaluated in performance tests. Anim. Sci. Pap. Rep., 28: 27-36.

$\mathrm{H}$ a u s b e r g e r M., M u 11 er C. (2002). A brief note on some possible factors involved in the reactions of horses to humans. Appl. Anim. Behav. Sci., 76: 339-344.

Hausberger M., Bruderer C., Le Scolan N., Pierre J.-S. (2004). Interplay between environmental and genetic factors in temperament/personality traits in horses (Equus caballus). J. Comp. Psychol., 118: 434-446.

H a u s b e rg e r M., M u 11 e r C., L u n e 1 C. (2011). Does work affect personality? A study in horses. PLoS One. Feb 9. 6: e14659.

H a y e s K.E.N. (1998). Temperament tip-offs. Horse Rider, 37: 46-51. 
Janczarek I., Kę dzierski W., S ta churska A., Wilk I. (2013 a). Emotional reactions of horses and trainers during natural method training. Ann. Anim. Sci., 13: 263-273.

Janczarek I., Stachurska A., Kę dzierski W., Wilk I. (2013 b). Responses of horses of various breeds to a sympathetic training method. J. Equine Vet. Sci., 33: 794-801.

J a n c z a r e k I., S t a c hurs k a A., Wi 1 k I. (2013 c). Kinematic parameters of the free jumping horse in the first stride of approaching the obstacle. Acta Agricult. Scand. Anim. Sci., DOI:10.1080/0906 4702.2013.785584.

K ę d zi ers ki W., J a n c z arek I., S t a c hurska A. (2012). Emotional response of naive Purebred Arabian colts and fillies to sympathetic and traditional training methods. J. Equine Vet. Sci., 32: $752-756$.

L 1 o y d A.S., M a r t in J.E., B or n e t t - G a u c i H.L.I., Wi 1 k in s o n R.G. (2008). Horse personality: variation between breeds. Appl. Anim. Behav. Sci., 112: 369-383.

M c Greevy P.D., Thom s on P.C. (2006). Differences in motor laterality between breeds of performance horse. Appl. Anim. Behav. Sci., 99: 183-190.

Murphy J., Arkin s S. (2007). Equine learning behaviour. Behav. Process., 76: 1-13.

S chmidt A., Aurich J., Möst 1 E., Muller J., A urich C. (2010). Changes in cortisol release and heart rate and heart rate variability during the initial training of 3-year-old sport horses. Horm. Behav., 58: 628-636.

T h a y e r J.F., L a n e R.D. (2009). Claude Bernard and the heart-brain connection: Further elaboration of a model of neurovisceral integration. Neurosci. Biobehav. Rev., 33: 81-88.

T h o m a s R.K. (1986). Vertebrate intelligence: a review of the laboratory research. In: Animal Intelligence: Insights into the Animal Mind, Hoage R.J., Goldman L. (eds). Smithsonian Institution Press, Washington DC.

Valenchon M., Lévy F., Prunier A., Moussu C., Cal andreau L., Lansade L. (2013). Stress modulates instrumental learning performances in horses (Equus caballus) in interaction with temperament. Plos One, 8: e62324.

Vis s e r E.K., Van Di erendonck M., E1lis A.D., Rijks e m C., Van Reenen C.G. (2009). A comparison of sympathetic and conventional training methods on responses to initial horse training. Vet. J., 181: 48-52.

War a n N., M c Gre ev y P., C a s e y R.A. (2007). Training methods and horse welfare. In: The Welfare of Horses. Springer, Netherlands, pp. 151-180.

Received: 7 VIII 2013

Accepted: 20 XII 2013 